\title{
DNAJC2 is reversely regulated by miR-627-3p, promoting the proliferation of colorectal cancer
}

\author{
HONGFU LIU ${ }^{1 *}$, JUAN LI $^{2 *}$, HUALI ZHAO ${ }^{3 *}$, XIAOHUI LIU ${ }^{4}$ and XINYING YE ${ }^{1}$ \\ ${ }^{1}$ Department of General Surgery, The First Affiliated Hospital of Gannan Medical University, Ganzhou, Jiangxi 341000; \\ ${ }^{2}$ Department of Dermatology and Venereal Diseases, Guiyang Center for Disease Control and Prevention, \\ Guiyang, Guizhou 550000; ${ }^{3}$ Department of Pediatric, The First Affiliated Hospital of Gannan Medical University, \\ Ganzhou, Jiangxi 341000; ${ }^{4}$ Department of Oncology, The First Affiliated Hospital of \\ Gannan Medical University, Ganzhou, Jiangxi 341000, P.R. China
}

Received January 28, 2021; Accepted May 24, 2021

DOI: $10.3892 / \mathrm{mmr} .2021 .12228$

\begin{abstract}
Colorectal cancer (CRC) is one of the most common malignancies among human, which is often connected with increased incidence and mortality rate. DnaJ Heat Shock Protein Family (Hsp40) Member C2 (DNAJC2) is an epigenetic factor, which is involved in a number of cytological functions, such as transcriptional regulation and ubiquitination. A number of studies reveal that DNAJC2 is closely associated with several tumors. However, the function and mechanism of DNAJC2 in CRC remains to be elucidated. In the present study, the expression of DNAJC2 was detected in CRC tissues and adjacent normal tissues. The results indicated that DNAJC2 was increased in CRC tissues and the expression level of DNAJC2 was significantly associated with tumor size. Cell function was detected via Cell Counting Kit-8, 5-ethynyl-2'-deoxyuridine, colony formation assays and flow cytometry by upregulating or knocking down of DNAJC2. Overexpression of DNAJC2 could accelerate cell proliferation while suppression of DNAJC2 decreased cell proliferation, possibly via the regulation of cell cycle regulation in vitro. It was also found that the function of DNAJC2 was reversely regulated by miR-672-3p, causing the promoting of cell proliferation through the activation of AKT/P21 signal pathway in CRC cells. These results suggested that DNAJC2 is a tumor-regulated protein in the progression of CRC and may represent a novel target for CRC detection and therapy.
\end{abstract}

Correspondence to: Dr Xinying Ye, Department of General Surgery, The First Affiliated Hospital of Gannan Medical University, 23 Qingnian Road, Ganzhou, Jiangxi 341000, P.R. China

E-mail: gupze12@163.com

*Contributed equally

Key words: colorectal cancer, DnaJ Heat Shock Protein Family (Hsp40) Member C2, microRNA, AKT, cell proliferation

\section{Introduction}

With the improvement of living environment, individual lifestyle is becoming increasingly westernized (1). Subsequently, the incidence and mortality rate from colorectal cancer (CRC) has also significantly increased. For instance, in China, CRC is included in the top five causes of cancer mortality (2). Similarly, CRC has the third-highest incidence rates among all types of cancer and it also ranks fifth in mortality caused by cancer in the US, in male and females (3). Thus, CRC might be a major threat to human health worldwide. One main reason for such high morbidity and mortality of CRC is lack of effective early diagnosis (4). Therefore, it has great significance for seeking new early tumor diagnostic markers.

DnaJ Heat Shock Protein Family (Hsp40) Member C2 (DNAJC2), also known as zuotin-related factor 1, was a recently identified epigenetic regulator of gene transcription in stem cells and cancer (5). DNAJC2 is located in the key region of 7q22-31.1 and high copy amplification of the gene has been reported to be involved in the progression of multiple types of cancer, such as prostate cancer, germ cell, glioblastoma, head and neck squamous cell carcinoma and gastric cancer (5-7). These data suggested that DNAJC2 is significantly related to proliferation and migration of tumor.

Little is known regarding the role of DNAJC2 in CRC, or its clinical and prognostic significance in patients with CRC. Therefore, the present study aimed to explore the expression level, biological function and potential mechanism of DNAJC2 in CRC.

\section{Materials and methods}

CRC patient specimens. A total of 84 pairs of human CRC tissues and normal colon ( $5 \mathrm{~cm}$ away from the tumor) or rectum mucosa adjacent ( $3 \mathrm{~cm}$ away from the tumor) to the tumor were acquired from patients after they underwent surgeries between September 2014 and April 2018 in The First Affiliated Hospital of Gannan Medical University. Clinical information was collected and presented in Fig. 1. The tissue samples were frozen in liquid nitrogen and preserved them in a refrigerator with a temperature of $-80^{\circ} \mathrm{C}$ as soon as possible until RNA extraction 
was performed. All of the enrolled patients or their relatives signed informed consent forms. The National Comprehensive Cancer Network (https://www.ncen.org/2015.2) was chosen for the identification of TNM. Patients cured by radiotherapy and chemotherapy were excluded from the present study. The present study was approved by the Ethics Committee of The First Affiliated Hospital of Gannan Medical University (approval no. IRB20170082) and was conducted in accordance with the Helsinki Declaration. The expression level of DNAJC2 in Oncomine database was obtained at https://www. oncomine.org.

Cell lines and culture conditions. CRC cell lines (LoVo, HCT-116, Caco-2, DLD-1 and HT-29), normal epithelial colon cells (NCM460) and 293T cells were purchased from the Cell Bank of the Chinese Academy of Sciences (Shanghai, China). Caco-2 were cultured in DMEM (Gibco; Thermo Fisher Scientific, Inc.); HCT-116 and HT-29 were cultured in McCoy's 5A medium (Invitrogen; Thermo Fisher Scientific, Inc.), DLD-1 was cultured in 1640 medium (Gibco; Thermo Fisher Scientific, Inc.), LoVo was cultured in F-12K medium (Gibco; Thermo Fisher Scientific, Inc.). All the media were supplemented with $10 \%$ FBS (WISENT Inc.) and $100 \mathrm{U} / \mathrm{ml}$ penicillin and $100 \mu \mathrm{g} / \mathrm{ml}$ streptomycin (WISENT Inc.). All the cells were cultured in a cell incubator with humid environment of $5 \% \mathrm{CO}_{2}$ and $37^{\circ} \mathrm{C}$ and all cell lines were authenticated using STR profiling.

Bioinformatical Analysis. In order to explore the detailed mechanism of DNAJC2 on CRC cell lines, online databases including TargetScan V3.1(http://www.targetscan. org/mamm_31/) and miRWalk 2021 (http://mirwalk.umm. uni-heidelberg.de/) were used to predict the potential miRNA for DNAJC2. The 3'untranslated region (UTR) was used as input to predict potential miRNA that may bind with DNAJC2.

$R N A$ extraction and reverse transcription-quantitative ( $R T-q)$ $P C R$. Total RNA, miRNA included, was extracted from tissues and cells by TRIzol reagent (Thermo Fisher Scientific, Inc.) in accordance with manufacturer's protocol and cDNA was produced by means of PrimeScript RT reagent kit (Takara Biotechnology Co., Ltd.). The RT kit was used according to the manufacturer's protocol. The RT-qPCR experiment was conducted through a $10 \mu \mathrm{l}$ reaction system which consists of $1 \mu \mathrm{l}$ cDNA, $1 \mu \mathrm{l}$ primers,

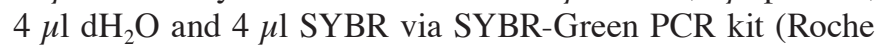
Diagnostics). StepOne Plus Real-time PCR system (Applied Biosystems; Thermo Fisher Scientific,Inc.) was used to performed final reaction. Thermocycling conditions was set as follows: Initial denaturation at $95^{\circ} \mathrm{C}$ for $3 \mathrm{~min}$, followed by 35 cycles at $95^{\circ} \mathrm{C}$ for $15 \mathrm{sec}, 60^{\circ} \mathrm{C}$ for $30 \mathrm{sec}$, and $72^{\circ} \mathrm{C}$ for $30 \mathrm{sec}$, followed by $72^{\circ} \mathrm{C}$ for $10 \mathrm{~min}$. The method used to standardize the RT-qPCR data was the $2^{-\Delta \Delta C a}$ method which facilitates the analysis of relative changes in gene expression in real-time quantitative PCR experiments (8). U6 was used as the internal controls for miRNA quantification while $\beta$-actin was used for mRNA. The primers used were: DNAJC2 forward, GTTGCGTTGTCTGCTTGAGGT; DNAJC2 reverse, CTGTGAATCTGTTCCCTGCTG; $\beta$-actin forward, AGCGAGCATCCCCCAAAGTT; $\beta$-actin reverse, GGGCACGAAGGCTCATCATT. Bulge-loop miRNA RT-qPCR Primer Sets specific for miR-672-3p were designed by Guangzhou RiboBio Co., Ltd.
Immunohistochemical assay. The tissue samples were fixed in $4 \%$ paraformaldehyde at $4^{\circ} \mathrm{C}$ overnight and sectioned. The tissue sections were mount on slides and were twice dehydrated on a rotor at room temperature over $10 \mathrm{~min}$ using a graded ethanol series: 15, 30, 50, 70, 90 and 100\%. After removing the paraffin and rehydration, the 5-mm thick sections were placed into a pressure cooker for $5 \mathrm{~min}$ to restore the antigen in the nucleus by using the citrate method (9). Then, $3 \% \mathrm{H}_{2} \mathrm{O}_{2}$ was used at room temperature for 5 min to suppress the endogenous peroxidase activity to reduce the background. The samples were blocked by $10 \%$ normal goat serum (WISENT, Inc.) and 5\% BSA (Beyotime Institute of Biotechnology) in TBS for $1 \mathrm{~h}$ at room temperature. The sections were incubated with primary antibody (1:400 dilution) overnight at $4^{\circ} \mathrm{C}$ and then washed three times in PBS (Beyotime Institute of Biotechnology). The primary antibodies used were DNAJC2, (cat. no. ab134572), Cyclin D1 (cat. no. ab134175), CDK2 (cat. no. ab235941), AKT (cat. no. ab18785), phosphorylated (p)-AKT (cat. no. ab38449), P21 (cat. no. ab188224) and $\beta$-actin (cat. no. ab179467). All the antibodies were purchased from Abcam. After incubation with HRP-conjugated secondary antibody (1:100 dilution; cat. nos. A0181, A0216 and A0208; Beyotime Institute of Biotechnology), sections were subjected to the DAB reaction (Beyotime Institute of Biotechnology). Images of the sections were captured by using a digital light microscope camera (Nikon Corporation). For each image, $\geq 5$ fields were randomly selected and presented with a magnification of $x 100$ and $x 400$. The positive area was analyzed using ImageJ 1.63 software (National Institutes of Health), as previously described (10).

Knockdown and overexpression of DNAJC2 and miR-672-3p. Small interfering RNA (siRNA) and negative control of DNAJC2 were Provided by Genomeditech. miR-672-3p mimics and inhibitors were supplied by Guangzhou RiboBio Co., Ltd. The sequence for miR-672-3p mimics was 5'-CCGATTCACCAACGA-3' and the control was 5'-TTTCATACATTCCAGC-3'. pLentiBlast-CMV-MCS-V5 1.2 vector was used for overexpression (GeneScript). The empty vector was applied for control group. Lipofectamine ${ }^{\circledR} 3000$ (Invitrogen; Thermo Fisher Scientific, Inc.) with $5 \mu \mathrm{g}$ plasmid (both control group and overexpression plasmid) was use for transfection in the DLD-1 and HCT-116 cell lines at room temperature overnight, with the density of $1 \times 10^{4} / \mathrm{ml}$. Cells were cultured for $6 \mathrm{~h}$ before changing the medium in cell incubator. After $48 \mathrm{~h}$, the efficiency of knockdown and overexpression assay was assessed via RT-qPCR detection.

Cell viability assay. To explore the effect of DNAJC2 on the proliferation of CRC cells, 96-well plates, each well containing $2 \times 10^{3}$ cells, were cultured in $100 \mu \mathrm{l}$ nutrient solution (Applied Biosystems; Thermo Fisher Scientific, Inc.). Cell Counting Kit-8 (CCK-8; Dojindo Molecular Technologies, Inc.) was used to detect the cell viability. A total of $10 \mu 1$ CCK- 8 was added to each well at 24, 48, 72 and $96 \mathrm{~h}$ and mixed with $90 \mu \mathrm{l}$ of serum-free medium per well. After incubation $\left(5 \% \mathrm{CO}_{2}\right.$ and $37^{\circ} \mathrm{C}$ ) for $2 \mathrm{~h}$, the absorbance value was measured by microplate spectrophotometer with wavelength set as $450 \mathrm{~nm}$.

5-Ethynyl-2'-deoxyuridine (EdU) assay. EdU assay kit (cat. no. C0071S; Beyotime Institute of Biotechnology) was used 
to measure cell proliferation. Cells were transferred into 96-well plates and cultured for $48 \mathrm{~h}$, each well containing $5 \times 10^{3}$ cells. Subsequently, EdU $(100 \mu \mathrm{l} ; 10 \mu \mathrm{M})$ was mixed with nutrient solution and incubated at $4^{\circ} \mathrm{C}$ for $3 \mathrm{~h}$. After that, cells were immersed in $4 \%$ paraformaldehyde at $4^{\circ} \mathrm{C}$ for $15 \mathrm{~min}$ and then treated with $0.3 \%$ Triton X-100 for $10 \mathrm{~min}$ at room temperature, which enhanced the permeability of the cells. Following washing with $3 \%$ BSA, a Nikon TI-DH light microscope (Nikon Corporation) was used to capture images at x100 magnification. In total, five independent visual fields were randomly selected for imaging, and ImageJ was used for optical density analysis.

Plate colony formation assay. Cells (500) were seeded into 6 -well plates in $37^{\circ} \mathrm{C}$. Every 5 days, each well was washed with PBS. After 10 days, cells were immersed into $0.2 \%$ crystal violet dye for staining at room temperature for $15 \mathrm{~min}$. Following washing with PBS, colonies ( $\geq 50$ cells/colony) in each well were manually counted via Photoshop CS6 (Adobe Systems, Inc.) and images were captured using a digital camera (Canon DS126211; Canon, Inc.).

Cell cycle distribution detection. First trypsin was used to digest the cells. The cells were centrifuged at $2,000 \mathrm{x} \mathrm{g}$ for $5 \mathrm{~min}$ at room temperature. Following washing with PBS, the cells were immersed into $75 \%$ ethyl alcohol and stored at $20^{\circ} \mathrm{C}$ overnight. Subsequently, the cells were washed with PBS again and stained with PI staining solution (300 $\mu$ l per Flow tube; Thermo Fisher Scientific, Inc.) for $10 \mathrm{~min}$ at room temperature in the dark, in accordance with the manufacturer's protocols. RNase was added for flow cytometry (Beyotime Institute of Biotechnology). The analysis was performed using a FACSCalibur flow cytometer (BD Biosciences) and CellQuest software (version 3.0; BD Biosciences).

Western blot analysis. A RIPA kit (Beyotime Institute of Biotechnology) was used to obtain the whole protein from cells and tissues. The protein concentration was measured via BCA Protein Assay kit (Beyotime Institute of Biotechnology). Then, 10\% SDS-PAGE was used for electrophoresis with $20 \mu \mathrm{g}$ protein in each lane. Protein was transferred onto polyvinylidene difluoride membranes (EMD Millipore). The membranes were blocked in $10 \%$ BSA solution (Beyotime Institute of Biotechnology) at room temperature for $2 \mathrm{~h}$ and incubated in an incubation box at $4^{\circ} \mathrm{C}$ with the primary antibodies overnight. The membranes then were washed with TBS with Tween-20 (5\% TBST) two or three times every 10-15 min. Subsequently, the membranes were incubated with immunoglobulin G (anti-mouse or rabbit) at room temperature for $2 \mathrm{~h}$ and washed with TBS $+0.001 \%$ Tween-20 buffer two or three times again, each time lasted 10-15 min. ECL Plus (EMD Millipore) was adopted for detection of protein expression levels by Bio-Imaging System. The primary antibodies included were: DNAJC2 (1:2,000; cat. no. ab231318), P21 (1:5,000; cat. no. ab109520; both Abcam), AKT (1:2,000; cat. no. 10176-2-AP) and p-AKT (1:3,000; cat. no. 66444-1-Ig; both from ProteinTech Group, Inc.). Secondary antibodies were goat anti-rabbit IgG (H+L; 1:5,000; cat. no. 111-035-003; Jackson ImmunoResearch Laboratories, Inc.).
Luciferase reporter assay. HCT-116 cells and 293T cells were planted into 24 -well plates $\left(5 \times 10^{3}\right.$ per well). The overexpressed plasmid vectors were designed and provided by Genomeditech and reporter gene plasmid or miR-672-3p mimics were transfected into cells via Lipofectamine ${ }^{\mathrm{TM}} 2000$. After 48 h, firefly or Renilla luciferase assay reagent were mixed with cell lysate in each group. A luciferase Reporter Gene Assay kit (cat. no. GM-040501A; Genomeditech) was adopted for assessing luciferase activity on an Infinite M1000 PRO microplate reader (Tecan Group, Ltd.).

Statistical analysis. Data were presented in forms of mean \pm standard deviation. GraphPad Prism 5.0 software (GraphPad Software Inc.) and SPSS 20.0 (IBM Corp.) were used to analyze the data. Paired Student's t-test was used for comparisons expression of DNAJC2 in paired clinical samples. Moreover, an unpaired Student's t-test was employed for analyzing difference in the other experiments. One-way ANOVA followed by Tukey's and repeated measures ANOVA followed by paired t-test with Bonferroni's correction were used to compare data sets which contained a number of groups. $\mathrm{P}<0.05$ was considered to indicate a statistically significant difference.

\section{Results}

DNAJC2 is upregulated in CRC and is associated with clinicopathological factors. The expression of DNAJC2 was measured by RT-qPCR in 84 pairs of CRC and normal tissues. The expression of DNAJC2 was higher in tumor tissues compared with normal tissues, in the cohort enrolled in The First Affiliated Hospital of Gannan Medical University and the data obtained from the GEO database (Fig. 1A and B). Similarly, the protein expression was also markedly higher in tumor tissues compared with normal tissues, which was confirmed by immunohistochemical analysis (Fig. 1C and D). Next, the association between DNAJC2 expression level and clinicopathological features of patients with CRC (Table I) was ascertained and the 84 cases of CRC tissue samples were divided into two experimental groups (high, $n=42$; low, $n=42$, medium as the cutoff). The expression level of DNAJC2 was significantly associated with tumor diameter. However, no significant association was found between DNAJC2 levels and other factors such as age, sex, carcinoembryonic antigen, depth of invasion or primary tumor site.

DNAJC2 regulates the proliferative capacities of $C R C$ cells in vitro. To detect the expression level of DNAJC2 in CRC cells, five CRC cell lines (HCT-116, LoVo, Caco-2, HT-29 and DLD-1) and the normal colon epithelial cell line (NCM460) were examined by RT-qPCR and western blotting. It was found that the mRNA and protein levels of DNAJC2 were significantly increased in the CRC cell lines compared with that in the NCM460 cells (Fig. 2A and B). Based on the endogenous expression of DNAJC2, the DLD-1 cell line was chosen for transfection with si-DNAJC2 while HCT-116 cell line was chosen for transfection with overexpression plasmid. According to the results of RT-qPCR and western blotting, the expression of DNAJC2 was markedly decreased 

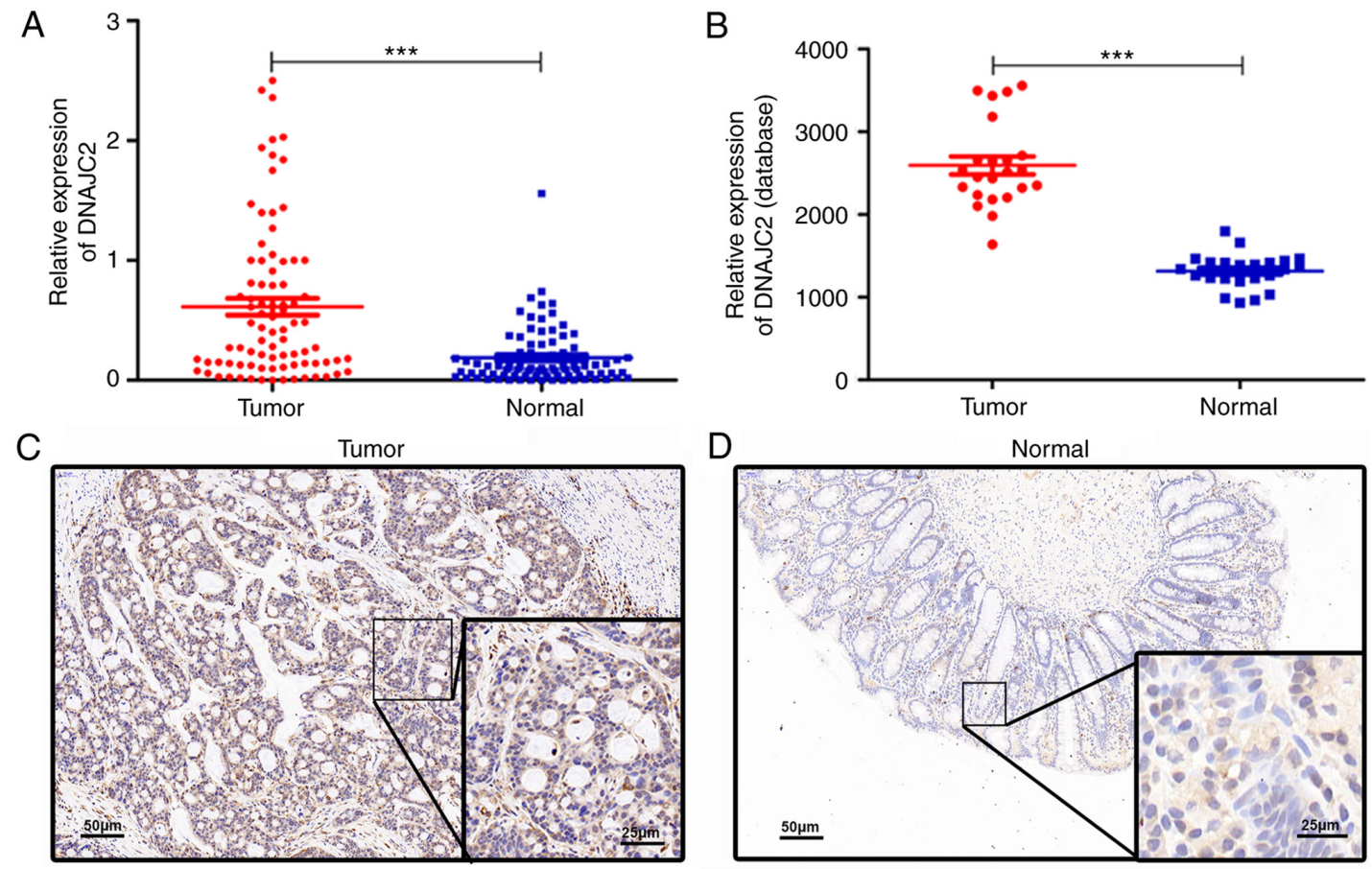

Figure 1. Expression of DNAJC2 in human CRC tissues. (A) mRNA expression levels of DNAJC2 in CRC and normal tissues (n=84) were detected by reverse transcription-quantitative PCR. mRNA levels of DNAJC2 were normalized to $\beta$-actin. (B) Data of DNAJC2 mRNA expression in CRC and normal tissues were retrieved from Oncomine database. Immunohistochemical results of DNAJC2 in (C) CRC tissues and (D) normal adjacent tissues. Images are displayed at x20 and x40 magnification, respectively, ${ }^{* * *} \mathrm{P}<0.001$. DNAJC2, DnaJ Heat Shock Protein Family (Hsp40) Member C2; CRC, colorectal cancer.

in DLD-1 cells (Fig. 2C and D) and was overexpressed by transfection with the DNAJC2-overexpressing plasmid in HCT116 cells (Fig. 2E and F). To further clarify the role of DNAJC2 in CRC, the DNAJC2-overexpressing plasmid and si-DNAJC2 were selected to perform further in vitro experiments.

Initially, it was hypothesized that DNAJC2 may serve an important role in CRC cell proliferation. Cells treated with si-DNAJC2 revealed markedly decreased proliferation compared with the control group (Fig. 3A). Colony-formation assay results also confirmed that DNAJC2 knockdown significantly reduced formation of colonies of DLD-1 cells (Fig. 3B). EdU and DAPI staining also showed that decreased expression of DNAJC2 suppressed the percentage of proliferative cells (Fig. 3C). Conversely, DNAJC2-overexpressing plasmid HCT-116 cells displayed prominently increased cell proliferation.

DNAJC2 influences CRC cell cycle progression to promote tumor growth. To clarify the role of DNAJC2 in CRC cell cycle progression, the cell cycle of DLD-1 and HCT-116 cell lines were analyzed with flow cytometry. It was observed that the DLD-1 cell line transfected with si-DNAJC2 apparently induced a $\mathrm{G}_{1}$ phase arrest compared with the control group. DNAJC2-overexpressing plasmid HCT-116 cells could also accelerate cell cycle progression (Fig. 4A and B). It was also discovered that increased DNAJC2 could promote the expression of cyclinD1 while attenuate the level of CDK2, otherwise, suppression of DNAJC2 could decease the level of cyclinD1 while promote CDK2 expression. (Fig. 4C). Therefore, the results showed that DNAJC2 might influence the cell cycle progression to promote the growth of CRC cells.
Table I. Association between DNAJC2 expression and the clinicopathological characteristics of patients with CRC.

\begin{tabular}{lcccc}
\hline Variable & $\mathrm{n}$ & $\begin{array}{c}\text { Low } \\
\mathrm{n}=42\end{array}$ & $\begin{array}{c}\text { High } \\
\mathrm{n}=42\end{array}$ & $\begin{array}{c}\text { P-value } \\
\chi^{2}\end{array}$ \\
\hline $\begin{array}{l}\text { Age (years) } \\
<60\end{array}$ & 32 & 18 & 14 & \\
$\geq 60$ & 52 & 24 & 28 & 0.833 \\
Sex & & & & \\
$\quad$ Male & 54 & 26 & 28 & \\
$\quad$ Female & 30 & 16 & 14 & 0.649 \\
$\quad$ Tumor diameter (cm) & & & & \\
$\quad<5$ & 42 & 26 & 16 & \\
$\geq 5$ & 42 & 16 & 26 & $0.029^{\mathrm{a}}$ \\
$\quad$ Lymph node invasion & & & & \\
$\quad$ Negative & 34 & 16 & 18 & \\
$\quad$ Positive & 50 & 26 & 24 & 0.657 \\
$\quad \begin{array}{l}\text { Depth of invasion } \\
\text { T1+T2 }\end{array}$ & 19 & 9 & 10 & \\
$\quad$ T3+T4 & 65 & 33 & 32 & 0.794 \\
Primary tumor site & & & & \\
$\quad$ Colon & 41 & 22 & 19 & \\
$\quad$ Rectum & 43 & 20 & 23 & 0.513 \\
CEA & & & & \\
$\quad<4.7$ & 40 & 22 & 18 & \\
$\quad 4.7$ & 44 & 20 & 24 & 0.382 \\
\hline
\end{tabular}

${ }^{\mathrm{a}} \mathrm{P}<0.05 ;$ DNAJC2, DnaJ Heat Shock Protein Family (Hsp40) Member C2; CRC, colorectal cancer; CEA, carcinoembryonic antigen. 
A

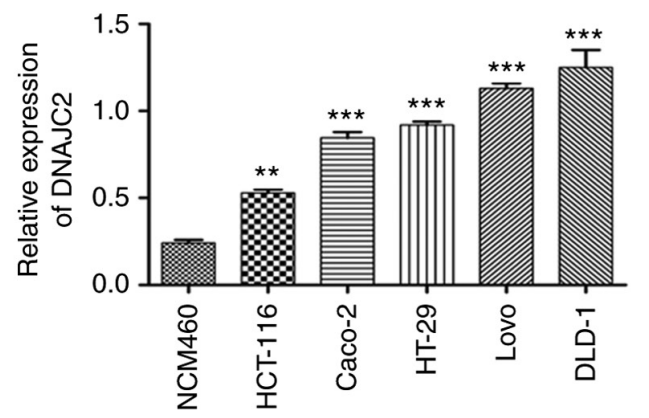

C

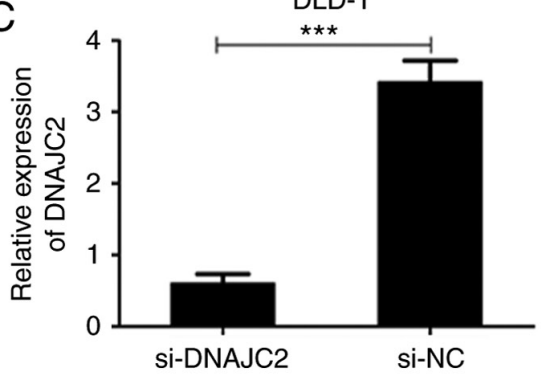

E

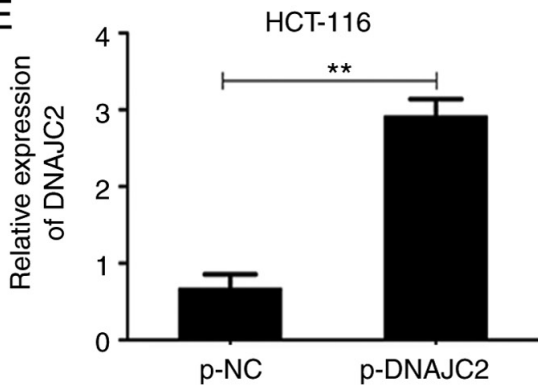

$\mathrm{B}$

DNAJC2

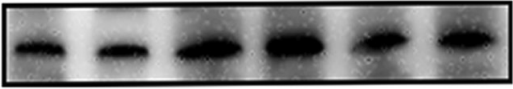

$\beta$-actin

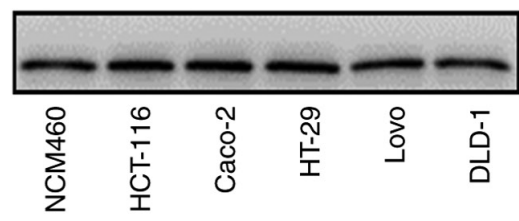

$\mathrm{D}$

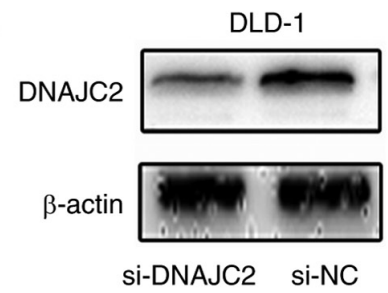

$\mathrm{F}$

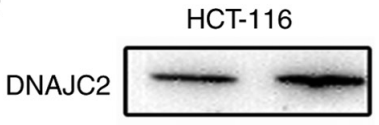

$\beta$-actin

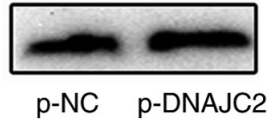

Figure 2. DNAJC2 is upregulated in CRC cell lines and could be regulated by siRNA and plasmid transfection. (A) mRNA expression of DNAJC2 in different CRC cell lines compared with NCM460 (normal colon epithelial cells). (B) Relative protein levels of DNAJC2 in different CRC cell lines compared with NCM460 normal colon epithelial cells. (C) Relative mRNA expression of DNAJC2 in DLD-1 cell line following the transfection of siRNA. (D) Relative protein levels of DNAJC2 in DLD-1 cell line following the transfection of siRNA. (E) Relative mRNA expression of DNAJC2 in HCT-116 cell line following plasmid transfection. (F) Relative protein levels of DNAJC2 in HCT-116 cell line following plasmid transfection. Data are presented as the mean \pm standard deviation. ${ }^{* *} \mathrm{P}<0.01$ and ${ }^{* * *} \mathrm{P}<0.001$. DNAJC2, DnaJ Heat Shock Protein Family (Hsp40) Member C2; CRC, colorectal cancer; siRNA, small interfering RNA

miR-672-3p reversely targets DNAJC2 through binding with the 3'UTR. In order to explore the detailed mechanism of DNAJC2 on CRC cell lines, online databases including TargetScan V3.1 (http://www.targetscan.org/mamm_31/) and miRWalk 2021 (http://mirwalk.umm.uni-heidelberg.de/) were used to predict the potential miRNA for DNAJC2. The 3'UTR region was used as input to predict potential miRNA that may bind with DNAJC2. miR-672-3p was predicted with the highest binding score according to the database (Fig. 5A and B). Next luciferase reporter assay was adopted to confirm the prediction. Mutant-type (MT) and wild-type (WT) DNAJC2 3'UTR sequences (site-directed mutations in the putative miR-672-3p target sites were included in the former) were cloned into reporter plasmids. The luciferase activity of DNAJC2 3'UTR was inhibited by over-expression of miR-672-3p. By contrast, the luciferase activity of DNAJC2-3'UTR-MUT was unaffected and the same phenomenon was also observed in HCT-116 cell lines (Fig. 5C). The above conclusions demonstrated that miR-672-3p is a potential upstream regulator of DNAJC2.

In CRC cell lines and the normal epithelial colon cell line the expression of miR-672-3p was downregulated in the
CRC cell lines compared with NCM460 (normal epithelial colon cell; Fig. 5D). In transfected CRC cells, RT-qPCR and western blotting demonstrated that expression level of DNAJC2 was lower in DLD-1 miR-672-3p-mimics and higher in HCT-116 miR-672-3p-inhibitor compared with the negative control (Fig. 5E-G). These findings indicated that miR-672-3p can directly negatively control DNAJC2.

miR-672-3p attenuates cell proliferation and regulates DNAJC2 through AKT/P21 pathway in CRC cell. The expression of miR-672-3p was next investigated in the same 84 patients with CRC. As presented in Fig. 6A, a downregulated level of miR-672-3p was observed in tumor tissues of patients with CRC. Meanwhile, the correlation of miR-672-3p and DNAJC2 in tissues samples was also analyzed and there was an inverse correlation between miR-672-3p and DNAJC2 (Fig. 6B).

To investigate whether miR-672-3p regulated cell proliferation, the endogenous expression of miR-672-3p in CRC cells lines was detected. The expression of miR-672-3p was relatively higher in HCT-116 and lower in DLD-1 (Fig. 6C). The function of miR-672-3p in CRC cells was next detected. CCK8 
A

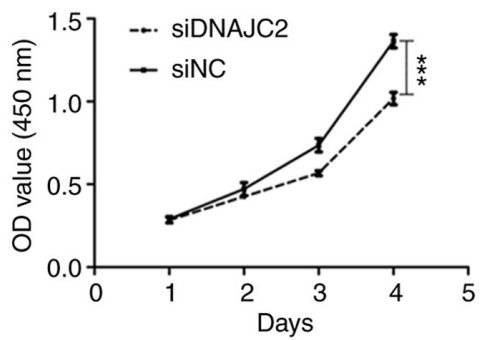

HCT-116

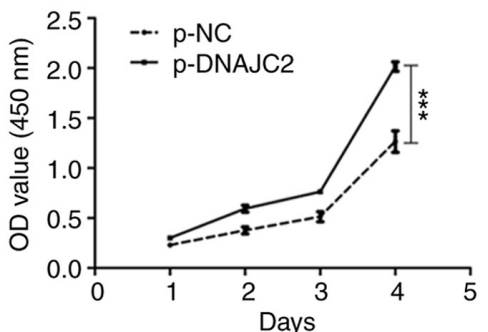

B

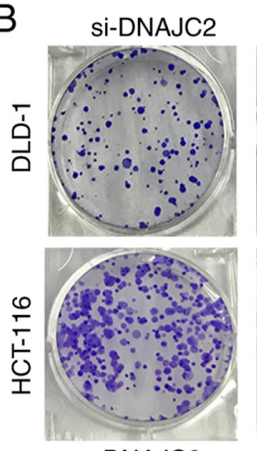

p-DNAJC2
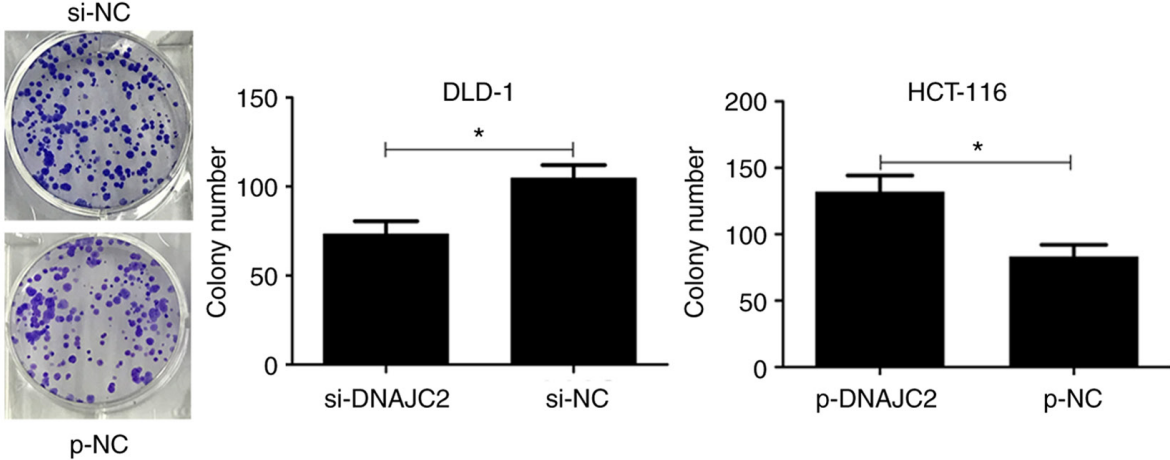

C
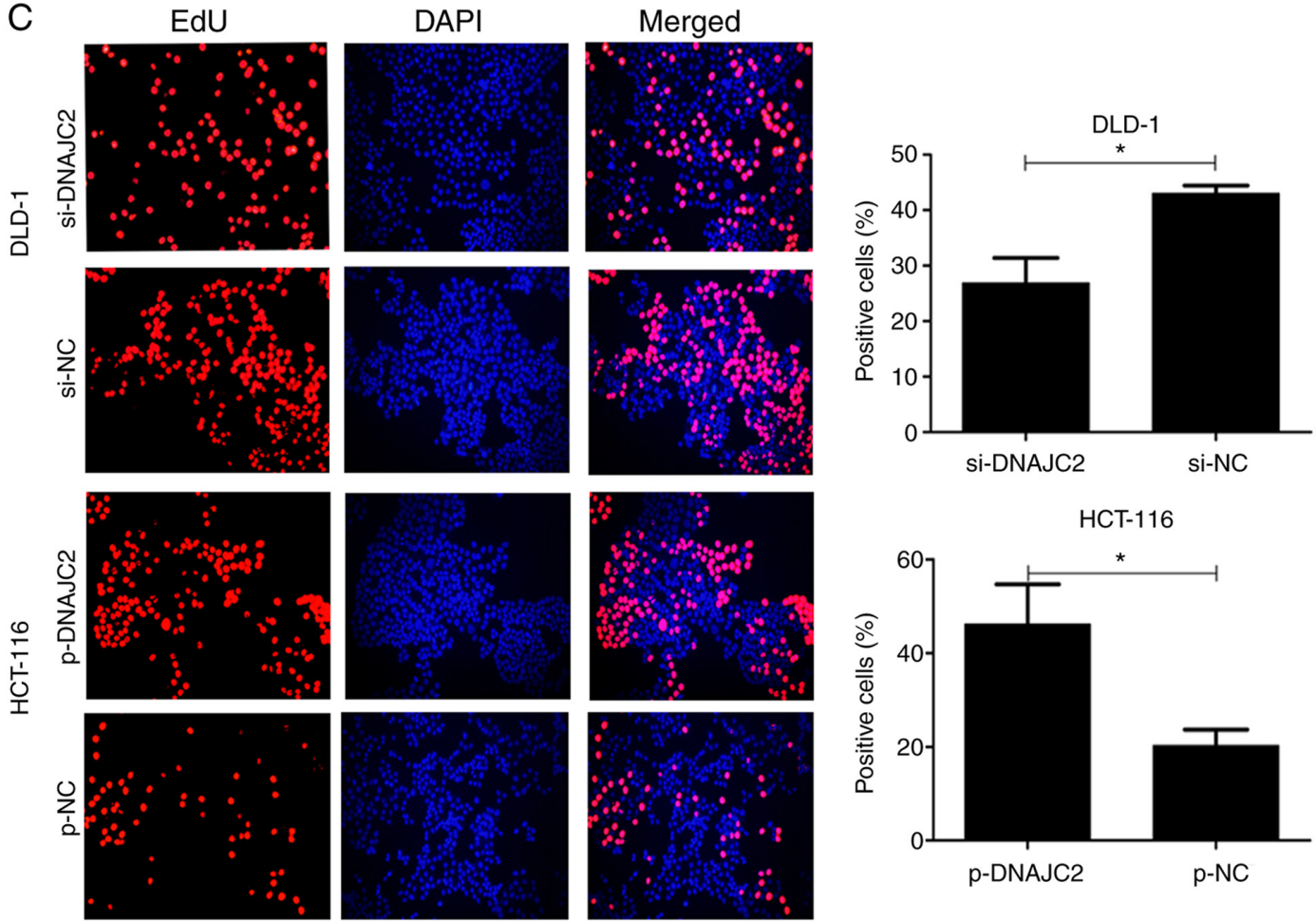

Figure 3. DNAJC2 promoted cell proliferation in CRC cells. (A) Cell counting kit-8 assays, (B) colony-formation ability and (C) EdU staining assays were adopted to detect the alteration of cell proliferation after the transfection of siRNA in DLD-1 cell line or following plasmid transfected into HCT-116 cell line. Presentative image was at x100 magnification. ${ }^{*} \mathrm{P}<0.05$ and ${ }^{* * *} \mathrm{P}<0.001$. DNAJC2, DnaJ Heat Shock Protein Family (Hsp40) Member C2; CRC, colorectal cancer; EdU, 5-ethynyl-2'-deoxyuridine; NC, negative control; p-, plasmid; siRNA, small interfering RNA.

and EDU assay were used for detecting the cell proliferation. It was found that increased miR-672-3p could attenuate the proliferation of CRC cells. Following miR-672-3p knockdown, cell growth could also be promoted (Fig. 6D and E). Cells overexpressed with miR-672-3p presented an increased $G_{0}-G_{1}$ and decreased $S$ phase percentage. The opposite result was observed with miR-672-3p knockdown (Fig. 6F).

The PI3K/AKT pathway serves an essential role in cell proliferation and cell cycle activities (11). Accordingly, P21 is positively correlated with malignancies by mediating cellular senescence $(12,13)$. The present study explored whether the PI3K/AKT pathway and P21 were closely connected to the cellular response which is mediated by DNAJC2 in CRC. Western blotting demonstrated decreased levels of p-AKT in the cells transfected with si-DNAJC2. The opposite phenomenon can be seen in the HCT-116 cell line, which was transfected by plasmid (Fig. 7A). The effects of miR-672-3p on CCND1, CDK2, p-AKT and P21 in CRC cells was also 

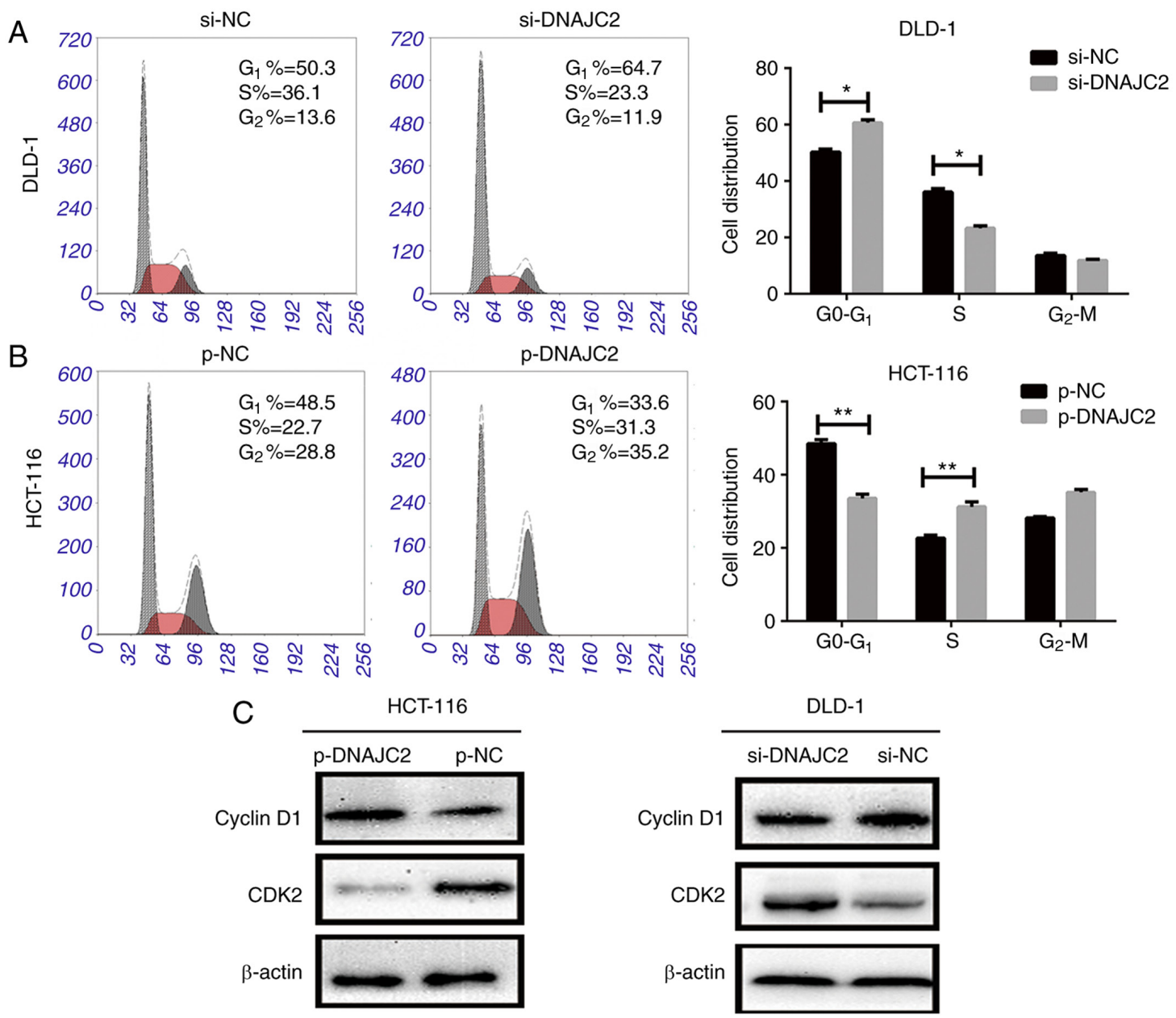

Figure 4. DNAJC2 regulates cell cycle in vitro. (A) In DLD-1 cell line the number of cells in $\mathrm{G}_{1}$ phase was prominently increased compared with the si-NC control following the transfection of siRNA. (B) Overexpression of DNAJC2 promoted the $\mathrm{G}_{1} / \mathrm{S}$ transition in HCT-116 cell line. (C) Key molecules of the $\mathrm{G}_{1}$ phase were detected by western blotting. Data are presented as the mean \pm standard deviation. ${ }^{*} \mathrm{P}<0.05$ and ${ }^{* *} \mathrm{P}<0.01$. DNAJC2, DnaJ Heat Shock Protein Family (Hsp40) Member C2; si, small interfering; NC, negative control; p-, plasmid.

investigated. It was found that cells overexpressed with miR-672-3p mimics suppressed Cyclin D1 while increasing CKD2 in CRC cell lines. The opposite result was observed following miR-672-3p knockdown. It was also found that cell treated with miR-672-3p mimics could decreased the phosphorylation of AKT with the accumulation of P21; however, when cells were treated with miR-672-3p inhibitor, the phosphorylation of AKT was increased accompanied by the suppression of P21 (Fig. 7B). According to the above results, it was concluded that DNAJC2 facilitates the proliferation of colorectal cancer via the p-AKT pathway.

\section{Discussion}

An increased level of DNAJC2 was found in tumor tissues as well as the CRC cell lines. It has been shown that DNAJC2 can accelerate transcriptional activation by particularly replacing polycomb-repressive complex 1 from chromatin at the beginning of differentiation (14). DNAJC2 has also been reported to be closely associated with different types of cancer. For example, in gastric cancer, DNAJC2 promotes cell proliferation, migration and invasion and induced apoptosis in a pathway mediated by p53 (15). In acute myeloid leukemia DNAJC2 can regulate the INK4-ARF locus during cellular proliferation and senescence (14). Knockdown of DNAJC2 can strongly inhibit leukemia progression by obtaining command of genes related to retinoic acid (RA) by means of its connection with the RA receptor $\alpha$ and its binding to genes relevant to RA, both of which describe the mechanism of DNAJC2 in acute myeloid leukemia at the molecular level (14). In breast cancer, DNAJC2 can be considered as potential molecular marker in the sera of patients with breast cancer (16). Another study verified that depletion of DNAJC2 promotes endocrine resistance via deregulation of cell death and cell survival related pathways (16). All these findings are consistent with the present study which indicated DNAJC2 function as a cancer promotor.

As already noted, increasing evidence demonstrates that miRNAs serve a crucial role in various biological processes and their unusual expression is implicated in a number of diseases, such as cancer and autoimmune disorders. miRNAs can act as a key role in several regulatory pathways involving CRC biology, such as epithelial-mesenchymal transition, angiogenesis and metastasis. For example, miR-1258 is a suppressive factor in a E2F8-mediated pathway. indicating that miR-1258 can be used as a therapeutic target for human CRC (17). Another study showed that miR-195-5p can downregulate the expression of YAP1 and miR-195-5p-mediated 


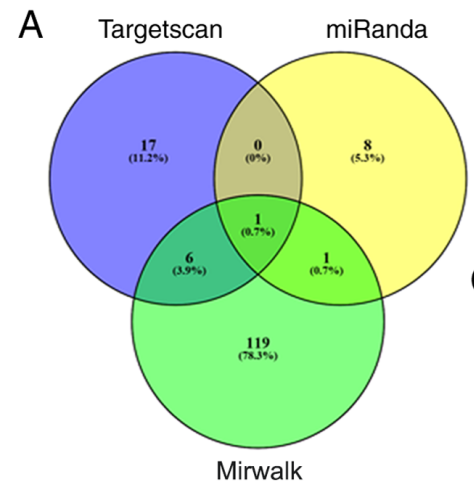

B

7:102977441-102977458

Position on chromosome: panTro2,rheMac2,oryCun2

Conserved species: (Transcript) 5' AGGGUAAGAUU C A $U$ C

GUG G C CA GGAAAAGG

Binding area: $\quad|| \mid$ | | | | |||||||

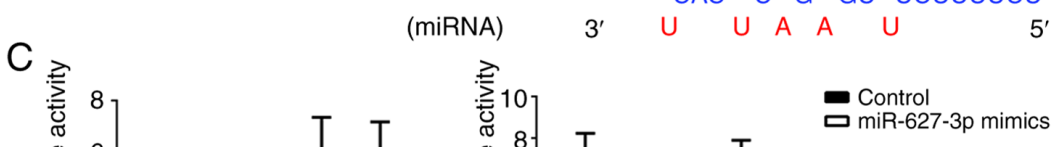
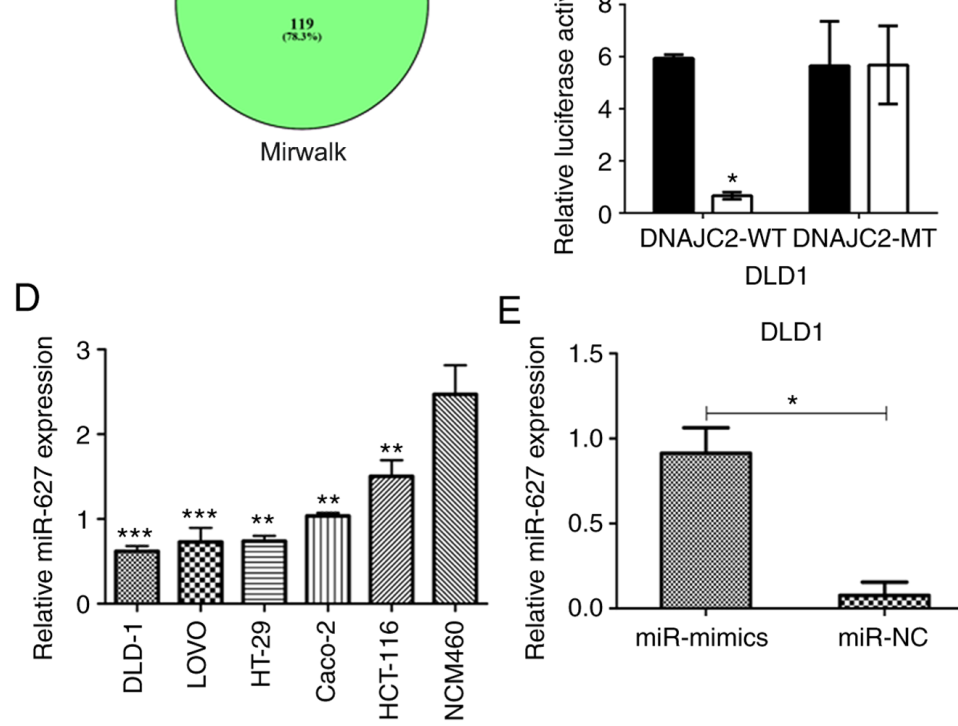

$\mathrm{E}$

DLD1
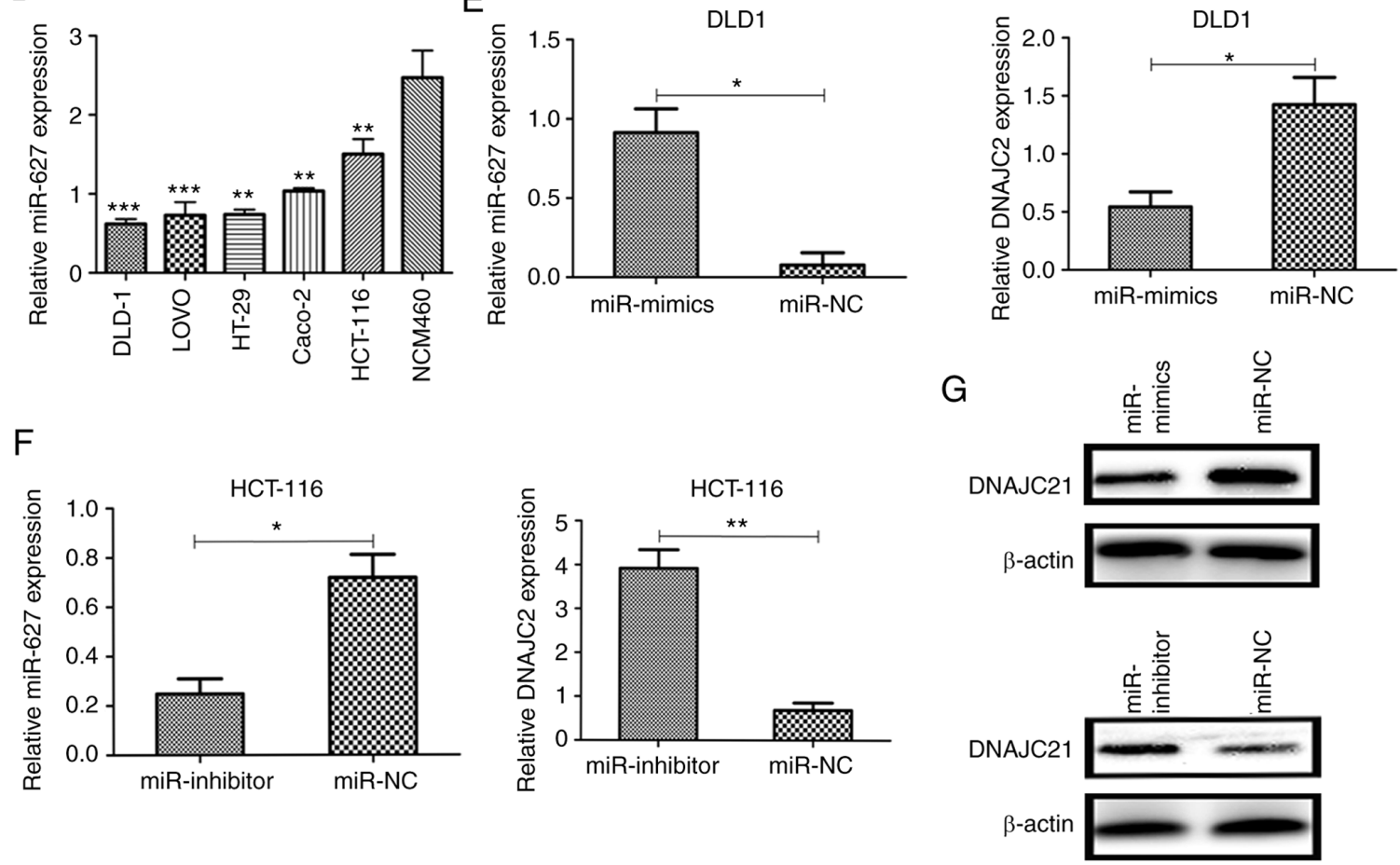

Figure 5. DNAJC2 is to be a potential target of miR-672-3p. (A) Three databases were used for forecasting of the potential upstream miRNAs of DNAJC2. (B) Binding site between DNAJC2 and miR-672-3p, as predicted by TargetScan. (C) Luciferase reporter assay was conducted to verify that miR-672-3p bound to the 3'-UTR region of DNAJC2 directly. (D) Expression of miR-672-3p was downregulated in five CRC cell lines compared with NCM460 normal colon cell line. Expression of DNAJC2 and miR-672-3p in (E) DLD-1 and (F) HCT-116 cell lines were detected by reverse transcription-quantitative PCR. (G) Expression of DNAJC2 and miR-672-3p in DLD-1 and HCT-116 cell lines were detected by western blotting. ${ }^{*} \mathrm{P}<0.05,{ }^{* *} \mathrm{P}<0.01$ and ${ }^{* * * *} \mathrm{P}<0.001$. DNAJC2, DnaJ Heat Shock Protein Family (Hsp40) Member C2; miR/miRNA, microRNA; CRC, colorectal cancer.

downregulation of YAP1, which impedes genesis and development of tumors in CRC (18). For miR-672-3p, it has been noted that miR-672-3p can serve a role of tumor suppressor in a number of types of cancer (19). However, its expression, function and mechanism in CRC remains to be elucidated. Overexpression of miR-672 could promote the angiogenesis of adipose-derived mesenchymal stem cells by suppressing tissue inhibitor of metalloproteinase 2 (20). miR-672 also induces osteoblast differentiation and mineralization through inhibition of Smad ubiquitin regulatory factor 1 with enhanced Runt-related transcription factor 2 transcriptional activation in mice (21). However, little has been reported on the function and mechanism in colorectal cancer.

Nevertheless, there are still a number of questions regarding the association between DNAJC2 and CRC remaining to be explored. First, as a transcription factor, the downstream targets of DNAJC2 need further examination. Second, whether miR-672-3p promotes or inhibits the development of colorectal cancer remains to be elucidated. Meanwhile, the role of DNAJC2 in invasion and metastasis of CRC is still unclear. Third, one of the limitations of the present study was not being able to provide results of in vivo studies it is hoped to provide more evidence with the help of a xenotransplantation model or other methods in future studies.

In conclusion, the present study verified that DNAJC2 can promote CRC cell proliferation and cell cycle progression by mediating cyclinD1, CDK2, p-AKT and p21 and miR-672-3p can regulate the expression of DNAJC2, all of which proved that DNAJC2 may serve a vital role as a potential therapeutic target of CRC. 
A
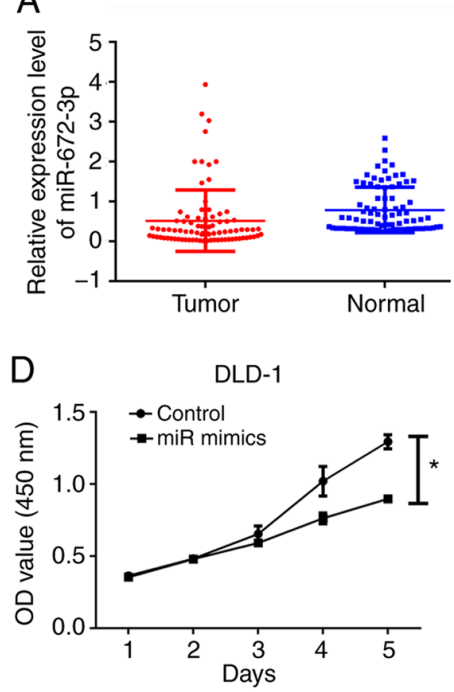

E

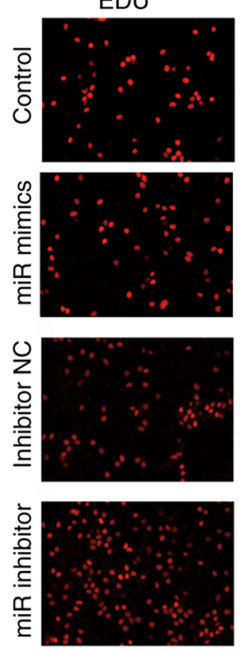

DAPI
B

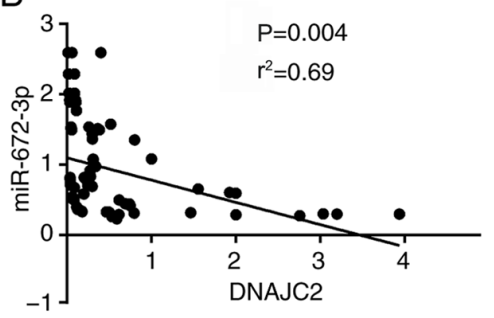

HCT-116
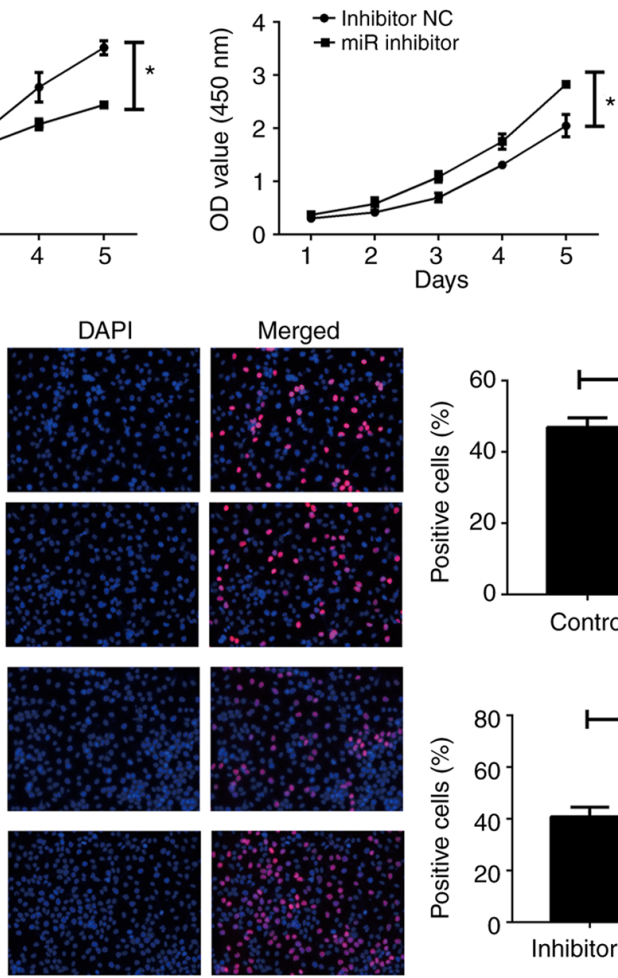

Merged
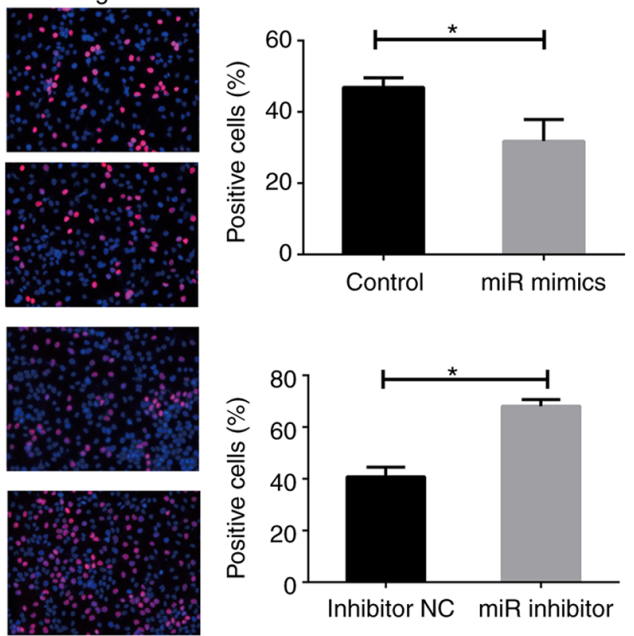
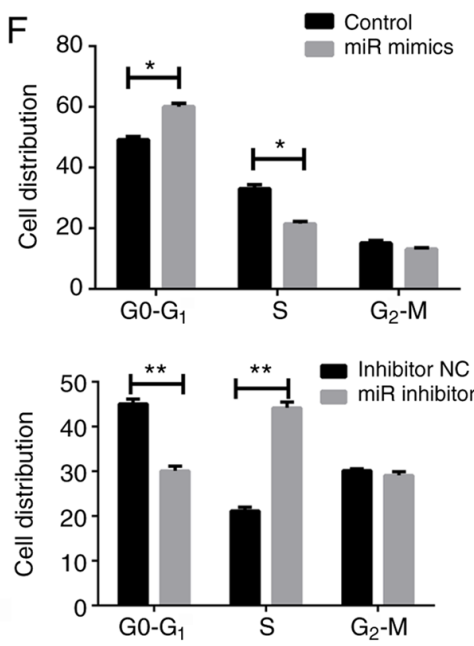

Figure 6. miR-672-3p reversely corrected with DNAJC2 and regulates cell proliferation and cell cycle in vitro. (A) Relative expression levels of DNAJC2 in CRC and normal tissues $(n=84)$. (B) Pearson correlation between miR-672-3p and DNAJC2. (C) Relative expression of miR-672-3p in different CRC cell lines compared with NCM460 (normal colon epithelial cells). (D) Cell proliferation was detected by CCK8 in cells treating with miR-672-3p mimics or miR-672-3p inhibitor. (E) Cell proliferation was detected by EdU in cells treating with miR-672-3p mimics or miR-672-3p inhibitor. Presentative image was at x100 magnification. (F) Cell cycle distribution of cells treating with miR-672-3p mimics or miR-672-3p inhibitor. " $\mathrm{P}<0.05$ and ${ }^{* *} \mathrm{P}<0.01$. miR/miRNA, microRNA; DNAJC2, DnaJ Heat Shock Protein Family (Hsp40) Member C2; CRC, colorectal cancer; EdU, 5-ethynyl-2'-deoxyuridine.
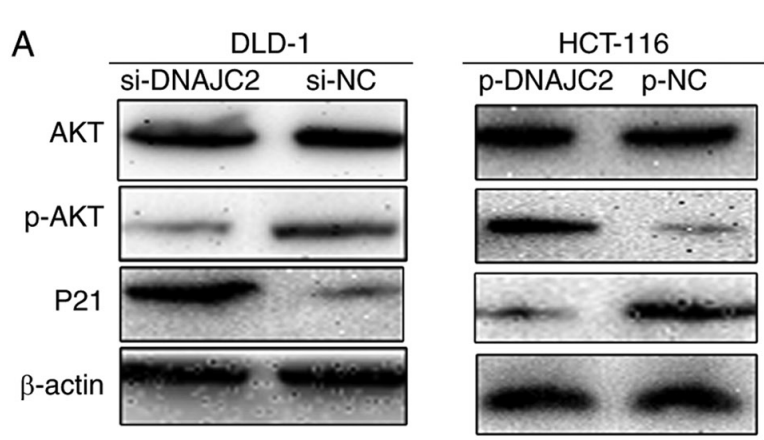

B

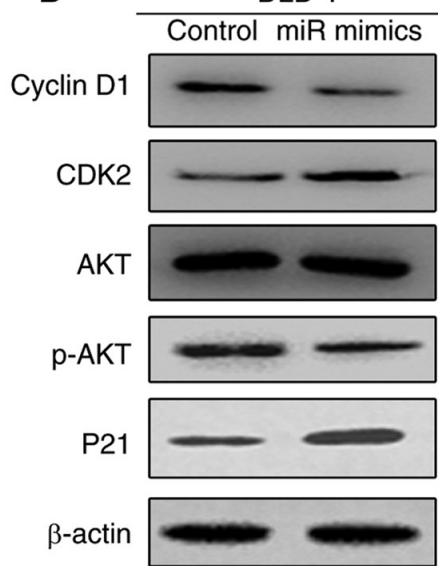

HCT-116

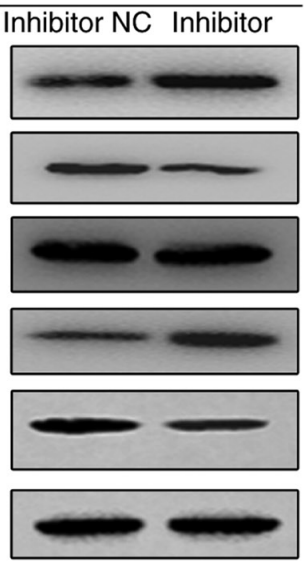

Figure 7. miR-672-3p and DNAJC2 influences the activation of the p-AKT pathways in CRC cells. (A) Expression of AKT, p-AKT and P21 was evaluated by western blotting in DLD-1 and HCT-116 cell lines compared with NC after the transfection of siRNA or plasmid. (B) Expression of Cyclin D1, CDK2, AKT, p-AKT and P21 was evaluated by western blotting in DLD-1 and HCT-116 cell lines after treating with miR-672-3p mimics or inhibitor. miR/miRNA, microRNA; DNAJC2, DnaJ Heat Shock Protein Family (Hsp40) Member C2; CRC, colorectal cancer; p-, phosphorylated; NC, negative control; si, small interfering. 


\section{Acknowledgements}

Not applicable.

\section{Funding}

The present study was funded by a grant of The First Affiliated Hospital of Gannan Medical University (grant no. GMU1011).

\section{Availability of data and materials}

The datasets used and/or analyzed during the current study are available from the corresponding author on reasonable request.

\section{Authors' contributions}

XY conceived the idea. HL designed experimental technology. HL and JL performed the majority of the experiments, analyzed and interpreted the results, produced figures and wrote the manuscript. HZ and XL performed the remainder of the experiments and analyzed the data. HL, JL, HZ and XL helped design the experimental studies and edited the manuscript. HL interpreted the results and wrote the manuscript. All authors reviewed and approved the final manuscript. HZ and XL confirm the authenticity of all the raw data.

\section{Ethics approval and consent to participate}

The present study was approved by the Ethics Committee of The First Affliated Hospital of Gannan Medical University (approval no. IRB20170082)

\section{Patient consent for publication}

Not applicable.

\section{Competing interests}

The authors declare that they have no competing interests.

\section{References}

1. Siegel RL, Miller KD, Fedewa SA, Ahnen DJ, Meester RGS, Barzi A and Jemal A: Colorectal cancer statistics, 2017. CA Cancer J Clin 67: 177-193, 2017.

2. Luo H, Zhang NQ, Huang J, Zhang X, Feng XL, Pan ZZ, Chen YM, Fang YJ and Zhang CX: Dietary intakes of different forms and sources of iron and colorectal cancer risk: A case-control study in China. Br J Nutr 121: 735-747, 2019.

3. Inadomi J and Jung B: Colorectal cancer-recent advances and future challenges. Gastroenterology 158: 289-290, 2020.

4. Dekker E, Tanis PJ, Vleugels JLA, Kasi PM and Wallace MB: Colorectal cancer. Lancet 394: 1467-1480, 2019.

5. Aloia L, Demajo S and Di Croce L: ZRF1: A novel epigenetic regulator of stem cell identity and cancer. Cell Cycle 14: 510-515, 2015.
6. Imamura T, Komatsu S, Ichikawa D, Miyamae M, Okajima W, Ohashi T, Kiuchi J, Nishibeppu K, Kosuga T, Konishi H, et al: Overexpression of ZRF1 is related to tumor malignant potential and a poor outcome of gastric carcinoma. Carcinogenesis 39: 263-271, 2018.

7. Demajo S, Uribesalgo I, Gutiérrez A, Ballaré C, Capdevila S, Roth M, Zuber J, Martín-Caballero J and Di Croce L: ZRF1 controls the retinoic acid pathway and regulates leukemogenic potential in acute myeloid leukemia. Oncogene 33: 5501-5510, 2014.

8. Livak KJ and Schmittgen TD: Analysis of relative gene expression data using real-time quantitative PCR and the 2(-Delta Delta C(T)) method. Methods 25: 402-408, 2001.

9. Waldvogel HJ, Curtis MA, Baer K, Rees MI and Faull RL: Immunohistochemical staining of post-mortem adult human brain sections. Nat Protoc 1: 2719-2732, 2006.

10. Varghese F, Bukhari AB, Malhotra R and De A: IHC Profiler: An open source plugin for the quantitative evaluation and automated scoring of immunohistochemistry images of human tissue samples. PLoS One 9: e96801, 2014.

11. Huang Y, Lin L, Shen Z, Li Y, Cao H, Peng L, Qiu Y, Cheng X, Meng M, Lu D, et al: CEBPG promotes esophageal squamous cell carcinoma progression by enhancing PI3K-AKT signaling. Am J Cancer Res 10: 3328-3344, 2020.

12. Liu Y, Marin A, Ejlerskov P, Rasmussen LM, Prinz M and Issazadeh-Navikas S: Neuronal IFN-beta-induced PI3K/Akt-FoxA1 signalling is essential for generation of FoxA1+Treg cells. Nat Commun 8: 14709, 2017.

13. Liu P, Begley M, Michowski W, Inuzuka H, Ginzberg M, Gao D, Tsou P, Gan W, Papa A, Kim BM, et al: Cell-cycle-regulated activation of Akt kinase by phosphorylation at its carboxyl terminus. Nature 508: 541-545, 2014.

14. Ribeiro JD, Morey L, Mas A, Gutierrez A, Luis NM, Mejetta S, Richly H, Benitah SA, Keyes WM and Di Croce L: ZRF1 controls oncogene-induced senescence through the INK4-ARF locus. Oncogene 32: 2161-2168, 2013.

15. Katsoulas A, Rachid Z, McNamee JP, Williams C and Jean-Claude BJ: Combi-targeting concept: An optimized single-molecule dual-targeting model for the treatment of chronic myelogenous leukemia. Mol Cancer Ther 7: 1033-1043, 2008.

16. Dyachenko L, Havrysh K, Lytovchenko A, Dosenko I, Antoniuk S, Filonenko V and Kiyamova R: Autoantibody response to ZRF1 and KRR1 SEREX antigens in patients with breast tumors of different histological types and grades. Dis Markers 2016: 5128720, 2016.

17. Zhang Z, Li J, Huang Y, Peng W, Qian W, Gu J, Wang Q, Hu T, $\mathrm{Ji}$ D, Ji B, et al: Upregulated miR-1258 regulates cell cycle and inhibits cell proliferation by directly targeting E2F8 in CRC. Cell Prolif 51: e12505, 2018

18. Liu X, Zhou Y, Ning YE, Gu H, Tong Y and Wang N: MiR-195-5p inhibits malignant progression of cervical cancer by targeting YAP1. OncoTargets Ther 13: 931-944, 2020.

19. Zierau O, Helle J, Schadyew S, Morgenroth Y, Bentler M, Hennig A, Chittur S, Tenniswood M and Kretzschmar G: Role of miR-203 in estrogen receptor-mediated signaling in the rat uterus and endometrial carcinoma. J Cell Biochem 119: 5359-5372, 2018

20. Chen M, Zhou M, Fu Y, Li J and Wang Z: Effects of miR-672 on the angiogenesis of adipose-derived mesenchymal stem cells during bone regeneration. Stem Cell Res Ther 12: 85, 2021.

21. Ahmad N, Kushwaha P, Karvande A, Tripathi AK, Kothari P, Adhikary S, Khedgikar V, Mishra VK and Trivedi R: MicroRNA-672-5p identified during weaning reverses osteopenia and sarcopenia in ovariectomized mice. Mol Ther Nucleic Acids 14: 536-549, 2019

This work is licensed under a Creative Commons Attribution-NonCommercial-NoDerivatives 4.0 International (CC BY-NC-ND 4.0) License. 